\title{
Patent ductus arteriosus ligation is associated with impaired left ventricular systolic performance in premature infants weighing less than $1000 \mathrm{~g}$
}

\author{
Patrick J. McNamara, MB Bch MRCPCH, ${ }^{\mathrm{a}, \mathrm{b}, \mathrm{c}}$ Lilian Stewart, MD, ${ }^{\mathrm{b}}$ Sandesh P. Shivananda, MD, \\ Derek Stephens, MSc, ${ }^{\mathrm{c}}$ and Arvind Sehgal, $\mathrm{MD}^{\mathrm{b}}$
}

Objective: Patent ductus arteriosus ligation is often complicated by systemic hypotension and oxygenation failure. The ability of the immature myocardium to compensate for altered afterload is poorly understood. The aim of this study was to characterize the effects of patent ductus arteriosus ligation on myocardial performance in preterm infants.

\begin{abstract}
Methods: Serial echocardiographic analysis was performed before and after patent ductus arteriosus ligation. Characteristics of the patent ductus arteriosus, myocardial performance (fractional shortening, mean velocity of circumferential fiber shortening, and left ventricular output) and left ventricular afterload (end-systolic wall stress) were assessed. The stress-velocity relationship was measured as a preload-independent, afterload-adjusted measure of myocardial contractility.

Results: Forty-six preterm infants were assessed at $28.5 \pm 11.3$ days and a weight of $1058 \pm 272 \mathrm{~g}$. Patent ductus arteriosus ligation was followed by increased left ventricular exposed vascular resistance temporally coinciding with reduced left ventricular preload, decreased left ventricular contractility, and low left ventricular output. Neonates weighing $1000 \mathrm{~g}$ or less had a higher rate of low fractional shortening $(<25 \%)$ or low left ventricular output $\left(<170 \mathrm{~mL} \cdot \mathrm{kg}^{-1} \cdot \mathrm{h}^{-1}\right)$ and increased need for cardiotropes and demonstrated a trend toward an impaired stress-velocity relationship. Neonates with impaired left ventricular systolic performance were more likely to require cardiotropes and have low systolic arterial pressure, increased heart rate, and abnormal base deficit.
\end{abstract}

Conclusion: Patent ductus arteriosus ligation is sometimes associated with impaired left ventricular systolic performance, which is most likely attributable to altered loading conditions. Neonates weighing $1000 \mathrm{~g}$ or less are at increased risk of impaired left ventricular systolic performance, which might relate to maturational differences and decreased tolerance to altered loading conditions. (J Thorac Cardiovasc Surg 2010;140:150-7)

Earn CME credits at

http://cme.ctsnetjournals.org

The hemodynamically significant ductus arteriosus (HSDA) is a common problem in premature infants that can lead to neonatal morbidity as a result of pulmonary overcirculation or systemic hypoperfusion. ${ }^{1-3}$ Failure of medical intervention occurs in $20 \%$ to $30 \%$ of extremely low-birth-weight infants, necessitating surgical intervention, ${ }^{4-6}$ although rates can vary between regions. Previous studies have demon-

\footnotetext{
From Physiology and Experimental Medicine, ${ }^{a}$ the Hospital for Sick Children Research Institute; and the Division of Neonatology ${ }^{\mathrm{b}}$ and the Department of Paediatrics, ${ }^{\mathrm{c}}$ University of Toronto, Toronto, Ontario, Canada.

Disclosures: None.

Dr Stewart is a coprincipal author.

Received for publication July 21, 2009; revisions received Dec 4, 2009; accepted for publication Jan 2, 2010; available ahead of print April 5, 2010.

Address for reprints: Patrick J. McNamara, MB Bch MRCPCH, the Hospital for Sick Children, 555 University Ave, Toronto, Ontario M5G 1X8, Canada (E-mail: patrick.mcnamara@sickkids.ca).

0022-5223/\$36.00

Crown Copyright (C) 2010 Published by Elsevier Inc. on behalf of The American Association for Thoracic Surgery

doi:10.1016/j.jtcvs.2010.01.011
}

strated that ligation of the patent ductus arteriosus (PDA) is complicated by hemodynamic instability and a need for escalation in respiratory support. ${ }^{7,8}$ We identified a relationship between age at ligation and risk of postligation cardiac syndrome, which is characterized by oxygenation failure and systolic hypotension requiring cardiotropic support within 8 to 12 hours of intervention. ${ }^{8}$ PDA ligation in premature baboons resulted in impaired left ventricular (LV) systolic performance and ventilation failure, which coincided with an increase in measured systemic vascular resistance (SVR). ${ }^{9}$ Because the neonatal myocardium is less tolerant of increased afterload, the risk of impaired LV systolic performance and clinical deterioration might be greater in more immature infants. ${ }^{10}$

Recently, impaired myocardial performance after PDA ligation, which is associated with altered preload, was demonstrated in neonates, ${ }^{11}$ but echocardiographic analysis was not performed during the period of clinical instability. The a priori primary aim of this study was to characterize the effect of PDA ligation on LV systolic performance and loading conditions. We hypothesized that PDA ligation is associated with impairment in LV performance coinciding with altered cardiac loading conditions during clinical deterioration. 


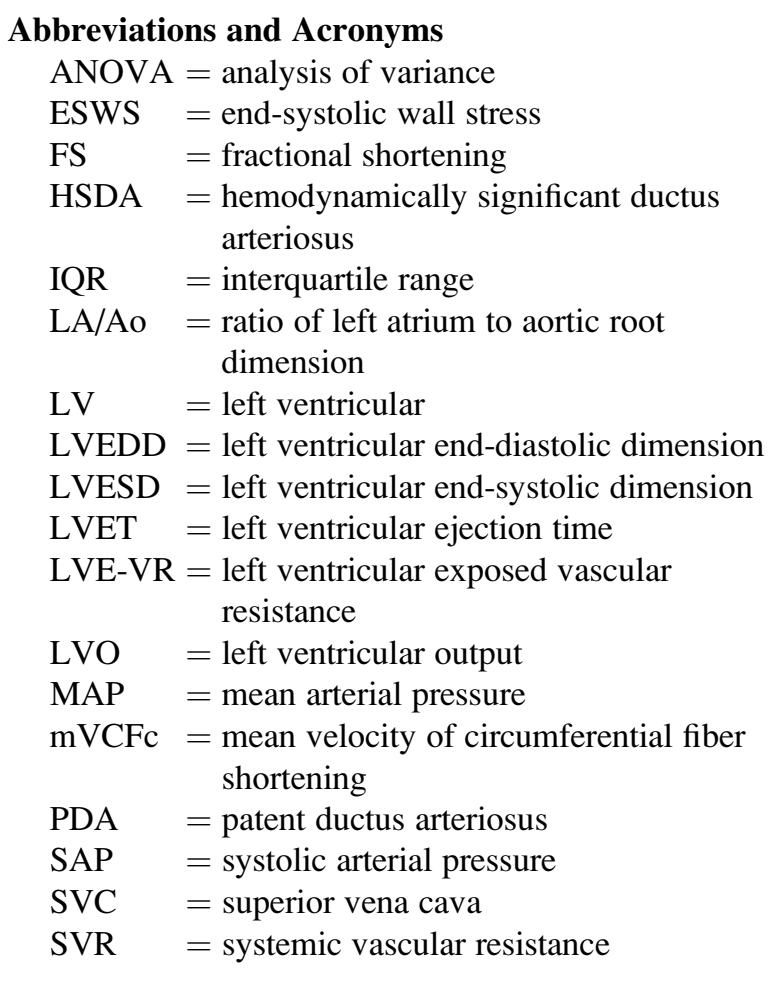

\section{MATERIALS AND METHODS}

We performed a prospective observational study performed at an outborn quaternary neonatal intensive care unit. The primary objective of this study was to characterize the effects of PDA ligation on LV systolic performance and loading conditions in premature infants. The a priori secondary objectives were to investigate the effect of these echocardiographic changes on cardiorespiratory well-being and determine whether neonates weighing less than $1000 \mathrm{~g}$ were at greater risk of impaired myocardial performance.

\section{Study Population}

Premature infants born at less than 32 weeks' gestation who underwent PDA ligation at the Hospital for Sick Children, Toronto, Ontario, Canada, between January and November 2005 were eligible for enrollment. Neonates were considered suitable for surgical ligation after failure of medical management or when indomethacin was contraindicated. The study population included neonates referred from 2 neighboring inborn tertiary care units and neonates from the study site, which is an outborn center. All patients were transported to the surgical site by a dedicated neonatal transport team at least 6 hours before the procedure. Newborns with congenital heart disease, except a patent foramen ovale, were excluded. The study was approved by the institutional research ethics board. Parental consent was obtained for all enrolled babies.

\section{Diagnosis of an HSDA}

An HSDA was clinically suspected when a murmur was associated with a wide pulse pressure, precordial activity, and cardiorespiratory instability or in the absence of a murmur caused by early-onset hypotension or oxygenation failure. Two-dimensional echocardiographic analysis was performed to quantify ductal characteristics before deciding on surgical ligation. An HSDA was defined by a transductal diameter of greater than $1.5 \mathrm{~mm}$ with unrestrictive $\left(<1.0 \mathrm{~m} \cdot \mathrm{s}^{-1}\right)$ left-to-right tranductal flow and clinical symptoms of pulmonary overcirculation, systemic hypoperfusion, or both.

\section{Medical Treatment}

The usual medical practice consisted of fluid restriction and 2 courses of high-dose $\left(0.2 \mathrm{mg} \cdot \mathrm{kg}^{-1}\right)$ indomethacin administered at 12-hour intervals unless adverse effects occurred or there was a contraindication to treatment. Prophylactic indomethacin $\left(0.1 \mathrm{mg} \cdot \mathrm{kg}^{-1}\right)$ was used for the first 3 days of life at one center.

\section{Preoperative Stabilization}

All patients had a hemoglobin level of greater than $100 \mathrm{~g} \cdot \mathrm{L}^{-1}$, a platelet count of greater than $100,000 / \mathrm{mm}^{3}$, and an international normalized ratio of less than 1.0 before surgical intervention. All neonates on high-frequency modes of ventilation were successfully switched to conventional ventilation, and enteral feeds were withheld for at least 6 hours before surgical intervention.

\section{Surgical Procedure}

The procedure was performed in the operating room by a cardiovascular surgeon. All neonates were anesthetized with fentanyl and either rocuronium or pancuronium. Newborns were placed in the right lateral position, and a left midaxillary incision was made. The left lung was retracted, and the ductus arteriosus was mobilized. The ductus was then either ligated with sutures or metal clips according to the surgeon. An intercostal drain was placed when there was a suspicion of pneumothorax. Fluid boluses $(0.9 \%$ saline) were administered for hypotension or excessive fluid losses.

\section{Routine Postoperative Care}

All neonates were transferred back to the neonatal intensive care unit and commenced on a morphine or fentanyl infusion. This was subsequently tapered over 6 to 24 hours depending on an objective assessment of pain using the Premature Infant Pain Profile score. ${ }^{12}$ A chest radiograph was obtained for all babies 1 hour after the procedure to assess lung inflation and exclude air leaks. Hypotension was defined by a mean arterial pressure (MAP) of less than that for corrected gestational age or a systolic arterial pressure (SAP) of less than the third percentile for corrected gestational age. Treatment decisions for cardiovascular instability were at the discretion of the attending neonatologist, who remained blind to all echocardiographic data. A fluid bolus of $10 \mathrm{~mL} \cdot \mathrm{kg}^{-1} 0.9 \%$ saline or cardiotropes was initially administered. The usual practice was to start with an infusion of dobutamine, dopamine, or both at 5 to 10 , which was titrated to a maximum of $20 \mu \mathrm{g} \cdot \mathrm{kg}^{-1}$ $\cdot \mathrm{min}^{-1}$, and escalate to an infusion of epinephrine, if necessary. Replacement steroid therapy with 1 to $2 \mathrm{mg} \cdot \mathrm{kg}^{-1}$ hydrocortisone was considered after failure of 2 cardiotropic agents.

\section{Data Collection}

Baseline neonatal characteristics, preligation neonatal morbidities, clinical and echocardiographic characteristics of the HSDA, dosage and duration of indomethacin treatment, and surgical details were reviewed. Physiologic indices of cardiorespiratory stability (eg, systolic and diastolic arterial pressures and oxygen saturation) were extracted from the electronic patient chart preoperatively and at 1, 4, 8, 12, and 24 hours after the procedure. MAP was measured directly by using a transducer connected to an indwelling catheter in the majority of patients because it is standard care at our institution to place a peripheral arterial line. In cases in which this was not feasible, noninvasive cuff readings were obtained by using the oscillometric method. Mean airway pressure (MAP) and fraction of inspired oxygen oxygenation index were monitored at these time points, as surrogates of the degree of respiratory support. The need for fluid boluses, blood transfusion, and cardiotropes was documented. 


\section{Study Protocol}

1. Two-dimensional echocardiographic analysis was performed preoperatively (within 2 hours of intervention) and at 1,8, and 24 hours after surgical intervention. Echocardiographic analysis was performed by a single operator (LS) who was trained in functional echocardiographic methodology under the direct supervision of the principal investigator (PJM). All evaluations were performed by using the Vivid 7 Advantage cardiovascular ultrasound system (GE Medical Systems, Milwaukee, Wis) with a 7.5-MHz high-frequency phased array transducer probe that allowed image acquisition at a rate of 400 frames per second. All images were saved to an optical disk for offline analysis.

2. For echocardiographic measurements/calculations, standard M-mode, 2-dimensional, pulsed-wave, continuous-wave, and color Doppler transthoracic echocardiographic analyses were performed.

For ductal assessment, baseline transductal diameter and flow pattern were calculated according to published methods. ${ }^{13}$

For LV systolic performance, left ventricular end-diastolic dimension (LVEDD), left ventricular end-systolic dimension (LVESD), and posterior wall thickness at end-diastole and peak systole were measured and averaged over 5 cycles. Left ventricular ejection time (LVET) was assessed by using M-mode tracings of aortic valve motion and measured from valve opening to closing. This was corrected to heart rate by dividing by the square root of the R-R interval (LVETc). LV fractional shortening (FS) was calculated as follows: (LVEDD - LVESD)/LVEDD. The mean velocity of circumferential fiber shortening $(\mathrm{mVCFc})$, a more accurate measure of contractility, was calculated as follows: FS/LVETc. Left ventricular output (LVO) was calculated as follows: [(Velocity time integral) $\times($ Heart rate $) \times$ (Aortic crosssectional area)] indexed to body weight. ${ }^{14}$ The stress-velocity relationship is a preload-independent, afterload-adjusted method of assessing LV contractility. ${ }^{15}$ The relationship between end-systolic wall stress (ESWS) and $\mathrm{mVCF}$ is evaluated according to linear regression methods. The slope of the regression line and linear intercept of the y-axis were then estimated for each time point. This relationship has been studied in full-term ${ }^{10}$ and premature infants both with and without a PDA. ${ }^{16} \mathrm{mVCFc}$ (circ/seconds) was calculated as follows: LV FS/LVETc. ESWS $\left(\mathrm{g} / \mathrm{cm}^{2}\right)$ was calculated as follows: $[1.35 \times(\mathrm{MAP}) \times(\mathrm{LVESD})] /[4 \times(\mathrm{HES}) \times(1+\mathrm{HES} / \mathrm{LVESD})]$, where 1.35 is the conversion factor from millimeters of mercury to grams per square centimeter, and HES is the LV posterior wall thickness at end systole. MAP was used because it has been shown to provide a reasonable estimate of LV end-systolic pressure in children. ${ }^{10}$ Because of the unreliability of LVO as an estimate of systemic blood flow in the presence of an HSDA, superior vena caval (SVC) flow was also calculated as follows: $[($ Velocity time integral $) \times($ Heart rate $) \times($ SVC cross-sectional area $)]$ indexed to body weight, as described by Kluckow and Evans, although there are no published norms for this postnatal age group.

LV preload was estimated from the LVEDD and the ratio of left atrium to aortic root dimension (LA/Ao ratio).

$\mathrm{LV}$ afterload is subject to the influence of vascular resistance; however, because the left ventricle is exposed to both pulmonary and systemic vascular beds before ductal closure, we calculated left ventricular exposed vascular resistance (LVE-VR) as a surrogate representation of the cumulative resistance. LVE-VR (dynes/s/ $\mathrm{cm}^{5}$ ) was calculated as follows: (MAP-Right atrial pressure)/LVO, with an estimated right atrial pressure value of $5 \mathrm{~mm}$ $\mathrm{Hg}$. ESWS $\left(\mathrm{g} / \mathrm{cm}^{2}\right)$ was also calculated as described above.

Pulmonary hemodynamics were assessed by calculating right ventricular systolic pressure $\left[4 \times(\text { Peak tricuspid regurgitant jet velocity })^{2}+5\right]$ and right ventricular output $[($ Velocity time integral $) \times($ Heart rate $) \times($ Pulmonary artery cross sectional area)] indexed to body weight.

Arterial blood gas testing was performed at baseline and 1, 8, and 24 hours after intervention.

\section{Outcome Measures}

The primary outcome studied was LV systolic performance, which was characterized by indices of LV contractility (FS and $\mathrm{mVCFc} / \mathrm{ESWS}$ relationship) and systemic blood flow (LVO). Indicators of impaired LV sys- tolic performance included an LV FS of less than $25 \%,{ }^{17,18}$ an LVO of less than $170 \mathrm{~mL} \cdot \mathrm{kg}^{-1} \cdot \min ^{-1},{ }^{17,19}$ an SAP of less than the third percentile, ${ }^{20}$ or a significant change from baseline in the slope of the $\mathrm{mVCFC} /$ ESWS regression line. Secondary outcomes included LV preload and afterload, heart rate, systolic and diastolic arterial pressure, arterial $\mathrm{pH}$, and base deficit.

\section{Statistics}

Descriptive statistics were used for neonatal demographics and characteristics of the ductus arteriosus, LV systolic performance, and perioperative hemodynamics. Repeated-measures analysis of variance (ANOVA) was used to analyze serial changes in indices of LV systolic performance, preload, and afterload. Linear regression analysis was used to determine the significance of correlations between $\mathrm{mVCF}$ and ESWS. Subgroup analysis was performed to compare neonates weighing less than $1000 \mathrm{~g}$ with those weighing more than $1000 \mathrm{~g}$ at surgical intervention because we speculated that smaller infants were at greatest risk of postoperative cardiorespiratory instability. Specifically, a comparison of the frequency of critical indicators of LV dysfunction (eg, LV FS $<25 \%$, LVO $<170 \mathrm{~mL} \cdot \mathrm{kg}^{-1} \cdot \mathrm{min}^{-1}$, and SAP less than the third percentile) was made between groups. Continuous variables were compared by using the $t$ test or Mann-Whitney $U$ test, respectively. Fisher exact and $\chi^{2}$ tests were used to analyze categorical variables. A comparison of the $\mathrm{mVCF} / \mathrm{ESWS}$ relationship was made at each respective time point. Analysis of covariance was used to compare the slopes and intercepts of the regression lines with baseline values. Subgroup analysis was performed between neonates with postoperative impaired LV systolic performance (LV FS $<25 \%$ or LVO $<170 \mathrm{~mL} \cdot \mathrm{kg}^{-1} \cdot \mathrm{min}^{-1}$ ) at any of the postoperative evaluation time points versus normal LV systolic performance to determine the clinical effect of these echocardiographic changes. We compared clinical markers suggestive of a low cardiac output state between groups and chose to focus on the first 8 hours after surgical intervention before the commencement of cardiotropic support. The average values for heart rate, arterial pressure, oxygen requirement, base deficit, and LVO were calculated. Univariate logistic analysis was also performed to identify predictors of LV FS of less than $25 \%$ and LVO of less than 170 $\mathrm{mL} \cdot \mathrm{kg}^{-1} \cdot \mathrm{min}^{-1}$. Data are presented as means \pm standard deviations or medians and interquartile ranges (IQRs). In this pilot study a convenience sample size of 40 patients was chosen.

\section{RESULTS}

Sixty-five neonates were referred for PDA ligation during the study period; parental consent was obtained for 46 babies. The mean gestational age and weight at birth were $25.3 \pm 1.4$ weeks and $777 \pm 212 \mathrm{~g}$, respectively. The average age at echocardiographic diagnosis of HSDA was 13.8 \pm 8.8 days. Indomethacin was administered to $86.9 \%$ patients, with patients receiving an average of $5.9 \pm 2.7$ doses. The mean postnatal age and weight at surgical intervention were $28.5 \pm 11.3$ days and $1058 \pm 272 \mathrm{~g}$, respectively. Transductal diameter was $3.6 \pm 0.4 \mathrm{~mm}$, with unrestrictive left-to-right transductal flow.

\section{Postoperative Cardiorespiratory Stability}

The postoperative period was characterized by a transient reduction in body temperature, decreased heart rate, and increased diastolic arterial pressure oxygen requirement and oxygenation index (Table 1). A postoperative increase in respiratory support was seen in $22(48 \%)$ patients. Three patients required postoperative high-frequency oscillation, all of whom weighed less than $1000 \mathrm{~g}$ and had impaired LV 
TABLE 1. Perioperative cardiorespiratory physiology and degree of respiratory support in neonates undergoing PDA ligation

\begin{tabular}{|c|c|c|c|c|c|c|c|}
\hline & Baseline & $1 \mathrm{~h}$ & $4 \mathrm{~h}$ & $8 \mathrm{~h}$ & $12 \mathrm{~h}$ & $24 \mathrm{~h}$ & $P$ value \\
\hline Heart rate (beats/min) & $154(148-163)$ & $146(139-161)$ & $144(135-155)^{*}$ & $148(142-157)$ & $150(140-161)$ & $150(142-160)$ & $<.001$ \\
\hline Temperature $\left({ }^{\circ} \mathrm{C}\right)$ & $36.8(36.6-37)$ & $36.4(36-36.9)$ & $36.5(36.4-36.8)^{*}$ & $36.7(36.4-36.9)$ & $36.8(36.5-37.1)$ & $36.8(36.7-37)$ & $<.001$ \\
\hline Systolic AP (mm Hg) & $55(51-64)$ & $58(51-63)$ & $54(48-61)$ & $54(47-63)$ & $53(49-59)$ & 57 (54-63) & .09 \\
\hline Diastolic AP (mm Hg) & $26(23-31)$ & $34(29-38)^{*}$ & $32(29-37)^{*}$ & $32(29-36)^{*}$ & $30(26-36)^{*}$ & $31(28-37)^{*}$ & $<.001$ \\
\hline $\mathrm{FIO}_{2}(\%)$ & $31(28-40)$ & $38(28-50)$ & $30(25-40)$ & $31.5(26-44)$ & $37(30-44)$ & $40(34-43)^{*}$ & .008 \\
\hline Oxygenation index & $7.5(5.4-9.9)$ & $7.4(5.0-9.3)$ & - & $8.1(5.9-14.5)$ & - & $9.7(7.3-13.6) \dagger$ & .02 \\
\hline $\mathrm{MAP}\left(\mathrm{cm} \mathrm{H}_{2} \mathrm{O}\right)$ & $8.8(8-10)$ & $9(8-10)$ & $8.7(8-9)$ & $9(8-10)$ & $8.9(8-10)$ & $9(8.1-10)$ & .85 \\
\hline $\mathrm{pH}$ & $7.34(7.29-7.37)$ & $7.36(7.31-7.41)$ & $7.32(7.24-7.41)$ & $7.31(7.27-7.37)$ & $7.29(7.27-7.34)$ & $7.32(7.29-7.36)$ & .048 \\
\hline $\mathrm{PaCO}_{2}(\mathrm{~mm} \mathrm{Hg})$ & $66(49-61)$ & $50(41-63)$ & $55.5(41-63)$ & $56(49-63)$ & $59.5(52-64)$ & $50(41-63)$ & .14 \\
\hline Base deficit & $3(-1$ to 6$)$ & $3(0$ to 4.5$)$ & $1(-2.8$ to 2.7$)$ & $2(-0.5$ to 4.5$)$ & $1.5(-1.5$ to 3.5$)$ & $1(-1.3$ to 3$)$ & .16 \\
\hline
\end{tabular}

Data are presented as medians (ranges). $P D A$, Patent ductus arteriosus; $A P$, arterial pressure; $F_{I O}$, fractional inspired oxygen; $M A P$, mean airway pressure. $* P<.05$. $\nmid P<.05$ vs 1 hour.

systolic performance before the transition. Perioperative cardiovascular support included crystalloid support $(\mathrm{n}=27$, $45 \%)$, red blood cell transfusion ( $\mathrm{n}=2,4.3 \%)$, cardiotropic support $(\mathrm{n}=8,17.4 \%)$, and hydrocortisone $(\mathrm{n}=2,4.3 \%)$ for cardiotrope-resistant shock. The median time for commencement of cardiotropic support was 10 hours (IQR, 6-12 hours).

\section{Postoperative Echocardiographic Analysis}

LV systolic performance. PDA ligation was associated with impaired LV systolic performance, as evident by a reduction in FS (Table 2) and mVCFc $(P<.001$, ANOVA; Figure 1 and Table 2) that was greatest 8 hours after surgical intervention. A significant decrease in $\mathrm{LVO}(P<.001$, ANOVA) and SVC flow index $(P=.001$, ANOVA) occurred, which was also maximal at 8 hours. An inverse correlation was identified between the ESWS and mean velocity of circumferential fiber shortening (stress-velocity relationship), but there were no differences in the slope or intercept values between preoperative and postoperative time points (Table 3).

LV preload/afterload. A marked reduction in LVEDD and the LA/Ao ratio $(P<.001$, Table 2$)$ was identified, paralle- ling the changes in LV systolic performance. PDA ligation was followed by an early and sustained increase in LVEVR $(P<.001$, Figure 2$)$, but there was no change in ESWS. Pulmonary hemodynamics. There were no changes in right ventricular systolic pressure; however, a significant decrease in right ventricular output occurred $(P<.001$, Table 2).

\section{Comparison of Neonates Weighing Less Than $1000 \mathrm{~g}$ $(\mathbf{n}=23)$ Versus More Than $1000 \mathrm{~g}(\mathrm{n}=\mathbf{2 3})$}

Neonates undergoing surgical ligation at a weight of less than $1000 \mathrm{~g}$ had lower gestational age (25 weeks [IQR, 2326 weeks] vs 26 weeks [IQR, 24-27 weeks], $P=.01)$ and weight at birth $(660 \mathrm{~g}$ [IQR, 500-820 g] vs $790 \mathrm{~g}$ [IQR, $555-1645 \mathrm{~g}], P=.001)$. There were no other demographic, duct-related, or anesthetic/surgical differences between groups. The rates of a postoperative critically low SAP of less than the third percentile, FS of less than $25 \%$, and LVO of less than $170 \mathrm{~mL} \cdot \mathrm{kg}^{-1} \cdot \mathrm{min}^{-1}$ were higher in neonates weighing less than $1000 \mathrm{~g}$ (Table 4). There was an inverse stress-velocity relationship in both subgroups, but there was no difference in the slope or intercept values (Table 3). In neonates weighing less than $1000 \mathrm{~g}$, the slope of the stress-velocity

TABLE 2. Perioperative echocardiographic characteristics of neonates undergoing PDA ligation

\begin{tabular}{|c|c|c|c|c|c|}
\hline & Baseline & $1 \mathrm{~h}$ & $8 \mathrm{~h}$ & $24 \mathrm{~h}$ & $P$ value \\
\hline LA/Ao ratio & $1.7 \pm 0.2$ & $1.3 \pm 0.2^{*}$ & $1.2 \pm 0.2 *$ & $1.3 \pm 0.2^{*}$ & $<.001$ \\
\hline LVEDD (mm) & $1.6(1.4-1.8)$ & $1.3(1.1-1.4)^{*}$ & $1.3(1.2-1.5)^{*}$ & $1.4(1.3-1.6)$ & $<.001$ \\
\hline LVESD (mm) & $0.9 \pm 0.2$ & $0.9 \pm 0.2$ & $0.9 \pm 0.2$ & $1.0 \pm 0.2$ & .11 \\
\hline LVPWD (mm) & $0.5(0.4-0.6)$ & $0.5(0.4-0.6)$ & $0.5(0.4-0.6)$ & $0.5(0.4-0.5)$ & .53 \\
\hline RVSP (mm Hg) & $7.9(6.5-12.4)$ & $7.4(6.7-12.9)$ & $7.8(6.6-11.7)$ & $10(6.8-16)$ & .73 \\
\hline LV FS $(\%)$ & $40(37-45)$ & $32(27-41)^{*}$ & $30(25-36)^{*}$ & $33(30-38)^{*}$ & $<.001$ \\
\hline SVC flow index $\left(\mathrm{mL} \cdot \mathrm{kg}^{-1} \cdot \mathrm{min}^{-1}\right)$ & $201 \pm 41$ & $164 \pm 37$ & $149 \pm 35^{*}$ & $179 \pm 28.7$ & .001 \\
\hline $\mathrm{RV}$ output $\left(\mathrm{mL} \cdot \mathrm{kg}^{-1} \cdot \min ^{-1}\right)$ & $692(498-1084)$ & $396(324-517)^{*}$ & $367(278-487)^{*}$ & $472(394-543)^{*}$ & $<.001$ \\
\hline LV output $\left(\mathrm{mL} \cdot \mathrm{kg}^{-1} \cdot \min ^{-1}\right)$ & $509(400-542)$ & $247(192-289)^{*}$ & $216(170-345)^{*}$ & $318(254-384)^{*}$ & $<.001$ \\
\hline $\mathrm{mVCFc}(\operatorname{circ} / \mathrm{s})$ & $1.6 \pm 0.4$ & $1.3 \pm 0.3^{*}$ & $1.3 \pm 0.3 *$ & $1.3 \pm 0.3^{*}$ & $<.001$ \\
\hline ESWS $\left(\mathrm{g} / \mathrm{cm}^{2}\right)$ & $21.6(18.1-30.7)$ & $21.1(14.2-26.1)$ & $22.4(16-28.7)$ & $23.1(20.1-34.2)$ & .07 \\
\hline
\end{tabular}

Data are presented as means \pm standard deviations or medians (ranges). $P D A$, Patent ductus arteriosus; $L A / A o$, left atrium/aortic ratio; $L V E D D$, left ventricular end-diastolic dimension; $L V E S D$, left ventricular end-systolic dimension; $L V P W D$, left ventricular posterior wall dimension; $L V F S$, left ventricular fractional shortening; $S V C$, superior vena cava; $R V$, right ventricular; $L V$, left ventricular; $m V C F c$, mean velocity of circumferential fiber shortening; $E S W S$, end-systolic wall stress. ${ }^{*} P<.05$. 


\section{LV exposed Vascular Resistance}

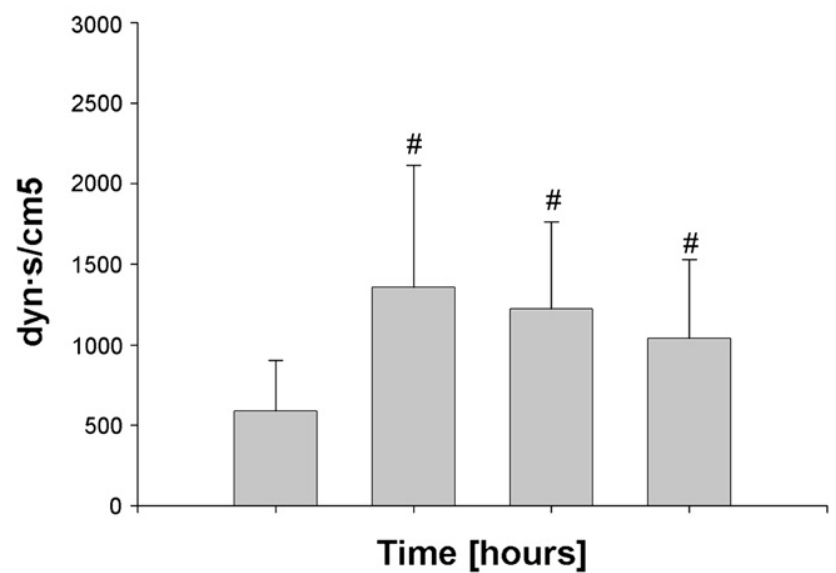

FIGURE 1. Trends in mean velocity of circumferential fiber shortening $(m V C F c)$ after patent ductus arteriosus ligation. $\# P<.05$ versus baseline, analysis of variance.

relationship at 8 hours trended $(P=.07)$ toward significance when compared with the preoperative value. In the subgroup of neonates weighing more than $1000 \mathrm{~g}$, there were no differences in either the slope or intercept between preoperative and postoperative stress-velocity relationship values.

\section{Comparison of Neonates With Impaired Versus Normal LV Performance}

For more information, see Table 5. Although neonatal demographics and ductal characteristics were comparable between groups, weight at surgical intervention was lower in neonates with low LVO. There were no differences in neonatal characteristics or amount of perioperative sedation/analgesia between groups. Neonates with impaired LV systolic performance had a higher heart rate and lower SAP and were more likely to have a higher base deficit in the first 8 hours after intervention. In addition, a higher proportion of these patients were treated with cardiotropic support.

TABLE 3. Stress-velocity relationship for all patients (neonates weighing $\leq 1000 \mathrm{~g}$ and $>1000 \mathrm{~g}$ )

\begin{tabular}{|c|c|c|c|c|}
\hline & Baseline & $1 \mathrm{~h}$ & $8 \mathrm{~h}$ & $24 \mathrm{~h}$ \\
\hline \multicolumn{5}{|c|}{ mVCFc/ESWS (all neonates) } \\
\hline Slope & -0.01 & -0.01 & -0.02 & -0.01 \\
\hline Intercept & 1.86 & 1.76 & 1.68 & 1.7 \\
\hline$R^{2}$ & -0.39 & -0.48 & -0.6 & -0.41 \\
\hline \multicolumn{5}{|c|}{$\mathrm{mVCFc} / \mathrm{ESWS}(<1000 \mathrm{~g})$} \\
\hline Slope & -0.015 & -0.013 & $-0.03 *$ & -0.013 \\
\hline Intercept & 1.83 & 1.73 & 1.94 & 1.7 \\
\hline$R^{2}$ & -0.36 & -0.37 & -0.65 & -0.37 \\
\hline \multicolumn{5}{|c|}{$\mathrm{mVCFc} / \mathrm{ESWS}(>1000 \mathrm{~g})$} \\
\hline Slope & -0.01 & -0.02 & -0.01 & -0.02 \\
\hline Intercept & 1.93 & 1.17 & 1.37 & 1.12 \\
\hline$R^{2}$ & -0.46 & -0.6 & -0.6 & -0.53 \\
\hline
\end{tabular}

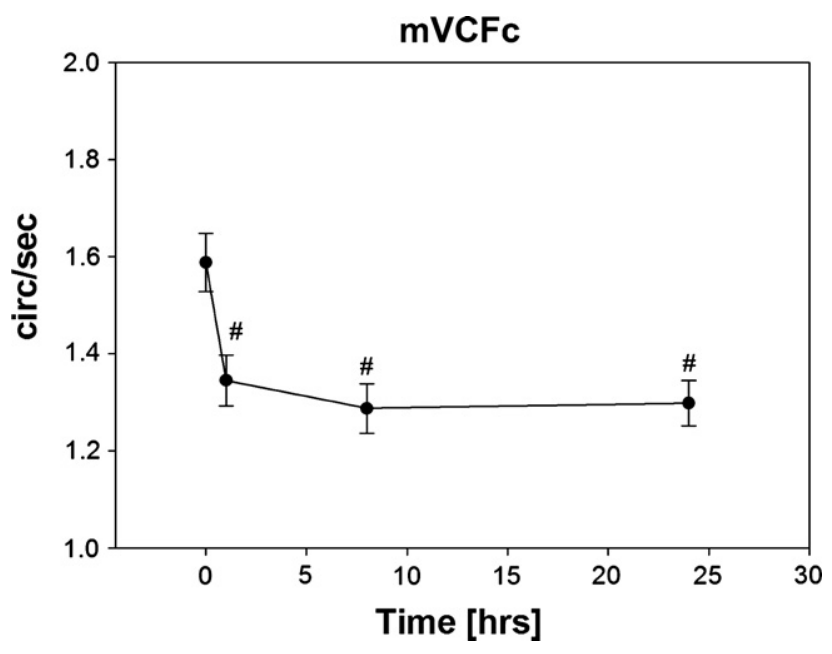

FIGURE 2. Trends in left ventricular $(L V)$ exposed vascular resistance after patent ductus arteriosus ligation. $\# P<.05$ versus baseline, analysis of variance.

\section{Predictors of LV Dysfunction}

Univariate analysis was performed to investigate any relationship between preligation or early postoperative factors and FS of less than $25 \%$ and LVO of less than $170 \mathrm{~mL}$. $\mathrm{kg}^{-1} \cdot \mathrm{min}^{-1}$ at 8 hours. Variables tested included clinical (birth weight, gestational age, preoperative diuretic or cardiotrope treatment, and age and weight at surgical intervention) and echocardiographic (transductal diameter, LA/Ao ratio, LVEDD, FS, mVCFc, LVO, SVC flow, ESWS, and LVE-VR at baseline and 1 hour) factors and postoperative need for cardiotropes. Only weight at intervention $(P=.04)$ was associated with FS of less than $25 \%$, but important trends for baseline LVEDD $(P=.05)$ and 1-hour ESWS $(P=.06)$ were noted. Preoperative LVEDD $(P=.04)$ and $\mathrm{SAP}(P=.002)$ values were associated with LVO of less than $170 \mathrm{~mL} \cdot \mathrm{kg}^{-1} \cdot \mathrm{min}^{-1}$.

\section{DISCUSSION}

PDA ligation is associated with major adaptive changes in pulmonary and systemic hemodynamics leading to cardiorespiratory instability. We identified impaired LV systolic performance and low LVO in a proportion of premature infants after surgical intervention. These changes temporally coincided with both increased LV exposed vascular resistance and decreased preload, which suggests that altered myocardial loading conditions might be responsible for the cardiovascular deterioration. The effect of these changes was greatest in neonates weighing less than $1000 \mathrm{~g}$, whose rate of cardiotropes was $30 \%$. The clinical course and rate of cardiovascular support in our cohort is comparable with published data. ${ }^{7}$

\section{PDA Ligation and LV Dysfunction}

PDA ligation was associated with a reduction in LV FS and mVCFC. In a preterm baboon model LV FS was shown to deteriorate 6 to 12 hours after surgical intervention, 
TABLE 4. Comparison of numbers of neonates weighing $<1000 \mathrm{~g}$ versus those weighing $>1000 \mathrm{~g}$ with impaired left ventricular contractility and low cardiac output before (preoperative) and 24 hours after (postoperative) surgical intervention

$<1000 \mathrm{~g}(\mathrm{n}=23) \quad>1000 \mathrm{~g}(\mathrm{n}=23)$

Preoperative period $(<6 \mathrm{~h}$ before intervention)

$\begin{array}{lcc}\begin{array}{l}\text { Systolic AP }<\text { third } \\ \text { percentile, } \mathrm{n}(\%)\end{array} & 1(4) & 0(0) \\ \mathrm{LVO}\left(\mathrm{mL} \cdot \mathrm{kg}^{-1} \cdot \mathrm{min}^{-1}\right) & 475.5 \pm 139.2 & 484.8 \pm 158.6 \\ \text { FS }(\%) & 41.6 \pm 8.0 & 41.7 \pm 5.6 \\ \text { Transductal diameter }(\mathrm{cm}) & 0.29 \pm 0.05 & 0.31 \pm 0.07\end{array}$

Postoperative period (24-h time period after intervention)

$\begin{array}{lcc}\begin{array}{l}\text { Systolic AP }<\text { third } \\ \text { percentile, } \mathrm{n}(\%)\end{array} & 8(35)^{*} & 1(4) \\ \mathrm{LVO}<170 \mathrm{~mL} \cdot \mathrm{kg}^{-1} . & 7(30)^{*} & 1(4) \\ \quad \min ^{-1}, \mathrm{n}(\%) & 7(30)^{*} & 1(4) \\ \mathrm{FS}<25 \%, \mathrm{n}(\%) & 16(70) & 12(52) \\ \text { Volume }>10 \mathrm{~mL} / \mathrm{kg}, \mathrm{n}(\%) & 1(4) & 1(4) \\ \text { Blood transfusion, n }(\%) & 7(30)^{*} & 1(4) \\ \text { Cardiotropes, } \mathrm{n}(\%) & 1(4.3) & 3(13) \\ \text { Hydrocortisone, } \mathrm{n}(\%) & 14(61)^{*} & 6(26) \\ \mathrm{FIO}_{2} \text { increase }>20 \%, \mathrm{n}(\%) & \end{array}$

Data are presented as frequencies (percentages of total) where relevant. $A P$, Arterial pressure; $L V O$, left ventricular output; $F S$, fractional shortening; $\mathrm{FIO}_{2}$, fractional inspired oxygen. $* P<.05$ versus neonates weighing more than $1000 \mathrm{~g}$.

coinciding with a temporal increase in measured SVR. ${ }^{9} \mathrm{We}$ identified a similar increase in LV exposed vascular resistance, coinciding with both impaired LV systolic performance and clinical deterioration. The left ventricle is exposed to the low-resistance pulmonary circuit in the presence of a patent ductus. ${ }^{20}$ Normalization of these conditions will occur after PDA ligation, leading to a net increase in vascular resistance, which might affect LV loading conditions. We made the assumption that the calculated LV ex- posed vascular resistance was a surrogate of the resistance the LV sees in the presence of a ductus. This assumption is limited because to accurately calculate this value, one would to have to apply the following formula for resistance circuits in parallel: Total resistance $=1 /(1 / \mathrm{R} 1+1 / \mathrm{R} 2)$, where $\mathrm{R} 1$ is $\mathrm{SVR}$ and R2 is the sum of peripheral vascular resistance and ductus arteriosus resistance. Despite these limitations, the magnitude of the change is likely to be important for a number of reasons. First, although ductal resistance cannot be measured in neonates, it is probably relatively low because of size and laminar flow change and unlikely to account for a 3-fold order of magnitude change in measured resistance. Second, postligation vascular resistance is above the normal range for human subjects (900-1200 dynes $\left.\cdot \mathrm{s}^{-1} \cdot \mathrm{cm}^{-5}\right)$. Finally the increase measured resistance is consistent with a previous report in human primates. ${ }^{9}$

Previous studies have failed to demonstrate any relationship between changes in LV systolic performance and afterload, ${ }^{21,22}$ although the study by Noori and colleagues ${ }^{11}$ suggested LV loading conditions to be important. Kimball and associates ${ }^{21}$ performed comprehensive echocardiographic assessments on 14 premature infants and concluded that although vascular resistance increased, myocardial performance did not change. Noori and colleagues ${ }^{11}$ studied 23 neonates and identified postoperative reductions in LVO and myocardial performance index, a surrogate of global myocardial function, which they attributed to decreased LV preload. These findings are important; however, both studies have some important limitations. First, the sample size is small and, in the latter study, includes a mixture of retrospective and prospective patients. Second, postoperative echocardiographic analysis was performed within 2 hours of intervention but not again until after 24 hours, missing the

TABLE 5. Comparison of neonatal demographics and morbidities, characteristics of the PDA, and status at surgical ligation in neonates with postoperative abnormal versus normal $L V$ performance

\begin{tabular}{|c|c|c|c|}
\hline Characteristic & Abnormal LV performance $(n=12)$ & Normal LV performance $(n=37)$ & $P$ value \\
\hline Gestation at birth (wk) & $25(24-26)$ & $25(24-26)$ & .75 \\
\hline Birth weight (g) & $700(650-948)$ & $730(640-787)$ & .98 \\
\hline PDA diagnosis (d) & $4(2-5)$ & $4(3-7)$ & .95 \\
\hline Transductal diameter $(\mathrm{mm})$ & $2.9(3.6-3)$ & $3(2.4-3.5)$ & .37 \\
\hline Indomethacin (doses) & $6(0-9)$ & $6(0-10)$ & .44 \\
\hline Age at ligation $(d)$ & $27 \pm 9.3$ & $29.9 \pm 12.1$ & .4 \\
\hline Weight at ligation $(\mathrm{g})$ & $891 \pm 161$ & $1096 \pm 283$ & .04 \\
\hline \multicolumn{4}{|l|}{ Postoperative status (n) } \\
\hline Heart rate $(1-8 \mathrm{~h})$ & $159(143-162)$ & $144(141-149)$ & .05 \\
\hline Respiratory rate $(1-8 \mathrm{~h})$ & $45 \pm 9.3$ & $35 \pm 8.3$ & .06 \\
\hline $\mathrm{FIO}_{2}(1-8 \mathrm{~h} ; \%)$ & $41 \pm 14.2$ & $35 \pm 11.5$ & .3 \\
\hline Systolic AP (1-8 h; mm Hg) & $44 \pm 7.9$ & $51.1 \pm 9.5$ & .04 \\
\hline Diastolic AP (1-8 h; mm Hg) & $32.9 \pm 6.3$ & $34.2 \pm 7.5$ & 6 \\
\hline Oxygenation index $(1-8 \mathrm{~h})$ & $11.8(7.4-13.1)$ & $7.7(5.6-10.8)$ & .1 \\
\hline Base deficit $<4.0(1-8 \mathrm{~h} ; \mathrm{n})$ & $5(56 \%)$ & $2(5.4 \%)$ & .007 \\
\hline Postoperative cardiotropes, $\mathrm{n}$ & $6(50 \%)$ & $2(5 \%)$ & .001 \\
\hline
\end{tabular}

Data are presented as means \pm standard deviations, medians (interquartile ranges), or frequencies (percentages of total) where relevant. $P D A$, Patent ductus arteriosus; $L V$, left ventricular; $\mathrm{FIO}_{2}$, fractional inspired oxygen; $\mathrm{AP}$, arterial pressure. 
time of clinical deterioration. We did not assess global myocardial performance, but we did identify a significant decrease in LVO, right ventricular output, and SVC flow. The changes in LVO were also demonstrated in 21 neonates studied by Lindner and coworkers. ${ }^{22}$ The finding of low LVO and impaired LV performance is clinically relevant because these patients had higher heart and respiratory rates and lower SAPs and were more likely to have an abnormal base deficit in the first 8 hours. In addition, a higher proportion of these patients subsequently required treatment with cardiotropic agents.

The changes in pulmonary and systemic blood flow were temporally associated with impaired LV systolic performance. The nature of altered LV systolic performance is likely to relate to cardiac loading conditions, although determining the relative contribution of each is difficult. PDA ligation was associated with both a decrease in LVEDD and LA/Ao ratio, surrogates of left heart preload, and increased $\mathrm{LV}$ exposed vascular resistance. It is not possible to prove whether the changes in preload are causal, although the magnitude of the reduction suggests they are likely to have some effect. We did not identify any major overall change in the stress-velocity relationship, which is surprising in the context of increased vascular resistance. The maintenance of wall stress at low levels might be explained by low end-systolic dimension, suggesting wall stress to be also preload dependant, ${ }^{11,21}$ therein raising doubt over its reliability in this setting. The low number of patients requiring cardiotropes might have biased against detecting any measurable change. Nevertheless, the slope of the line at 8 hours, particularly in neonates weighing less than $1000 \mathrm{~g}(-0.03)$, is much higher than normative data for healthy premature infants of less than 30 weeks' gestation $(-0.004$ to -0.007$)$ and those with a severely low systemic blood flow state $(-0.01),{ }^{22}$ suggesting an effect on myocardial performance.

\section{Dysfunction and Immaturity}

Previously, we have shown that patients who undergo PDA ligation at an earlier age are at greatest risk of postoperative hemodynamic instability. ${ }^{8}$ There is also evidence that gestational age and weight at birth are predictive of need for vasopressor treatment. ${ }^{7}$ Developmentally, the immature myocardium has been shown to exhibit a higher basal contractile state and a greater sensitivity to afterload changes. ${ }^{10,23,24}$ The intolerance of the immature myocardium to increased afterload might be attributable to differences in myofibrillar architecture or immaturity of receptor development or regulation. ${ }^{25}$ In this study a higher proportion of neonates weighing less than $1000 \mathrm{~g}$ had low FS of less than $25 \%$ and low LVO of less than $170 \mathrm{~mL} \cdot \mathrm{kg}^{-1}$. $\min ^{-1}$ within 8 hours of intervention, suggesting a developmental effect. Interestingly, the slope of the stress-velocity relationship was also highest in neonates weighing less than $1000 \mathrm{~g}$ at 8 hours and demonstrated an interesting trend when compared with the preoperative slope. This suggests that the study might have been underpowered to determine a true difference. On univariate analysis, an association between weight at intervention and FS of less than $25 \%$ at 8 hours was identified. Preoperative LVEDD and SAP values were associated with an LVO of less than $170 \mathrm{~mL} \cdot \mathrm{kg}^{-1}$. $\min ^{-1}$ at 8 hours. These factors might be important considerations for perioperative monitoring.

\section{Clinical Relevance of LV Dysfunction}

The magnitude of the changes in heart rate, arterial $\mathrm{pH}$, ventilation requirements, and fraction of inspired oxygen oxygenation index were minimal, and numbers of patients requiring cardiotropes were low in the overall study population. This is not an unexpected finding because the study population includes older patients who were clinically well in the postoperative period with minimal echocardiographic changes and those with cardiorespiratory compromise and echocardiographic evidence of low LVO. Nevertheless, it is meritorious and of benefit to health care professionals who are managing these patients to be aware of the clinical and echocardiographic changes in both patient subgroups in monitoring these patients postoperatively.

Neonates with impaired LV performance had lower SAPs and higher rates of abnormal base deficit and need for cardiotropes. The physiologic effects in a preterm baboon model of PDA ligation included oxygenation failure in the first 72 hours, with an improvement in lung compliance and pulmonary hemodynamics by 1 week after the procedure. ${ }^{26}$ We have not yet addressed the long-term respiratory outcomes in this study. The recent articles by Kabra and coworkers ${ }^{5}$ and Chorne and associates ${ }^{4}$ highlight associations between PDA ligation and increased risk of bronchopulmonary dysplasia, severe retinopathy of prematurity, and neurosensory impairment. Whether PDA ligation is causal or is merely a surrogate marker of a biological state with an increased risk of adverse outcome remains unknown. The increased risk of clinical deterioration in neonates with impaired LV systolic performance further emphasizes the need to characterize any possible relationship of this period of instability to important neonatal outcomes. Early identification of patients at increased risk of cardiorespiratory compromise requires prospective evaluation.

\section{Limitations}

It is possible that the administration of crystalloid therapy or cardiotropic agents confounded the final echocardiographic evaluation; however, earlier studies (1 and 8 hours) were performed before the commencement of any cardiotropes. The contribution of anesthetic agents to the hemodynamic changes is an important consideration, although it is difficult to quantify in the absence of a control group. An anesthetic effect appears less likely because it would be expected to present much earlier in the postoperative period. 
Second, patients who had impaired LV systolic performance did not receive a higher amount of anesthesia. Echocardiographic measurements are subject to interobserver or intraobserver variability of $10 \%$ to $15 \%$, but the magnitude of the hemodynamic changes seen suggest them to be real.

Third, the persistently high ratio of right ventricular output/LVO after PDA ligation is an unexpected finding. This most likely relates to ongoing left-to-right atrial shunting, but unfortunately, we did not document the size of the patent foramen ovale/atrial septal defect. In addition, it is not possible to quantify the magnitude of the flow or pressure differential across it, which might have provided valuable information regarding left atrial pressure or LV end-diastolic pressure. The fact that SVC flow accounted for a higher proportion of right ventricular output after PDA ligation might also be important. Neonates with an HSDA might have reversed or absent cerebral blood flow, as estimated by means of pulsed-wave Doppler scanning, which is restored after PDA ligation. These changes might also relate to operator-dependant factors because echocardiography-based estimations of cardiac output are limited ${ }^{4}$; however, invasive estimates are not possible in extremely low-birth-weight infants. Our study might have been underpowered to clearly determine the effect of afterload, as evidenced by the low number of neonates who required cardiotropes. Finally, there might be important and relevant changes in diastolic performance that were not addressed in this study.

\section{CONCLUSION}

The postoperative course after PDA ligation is variable, but in some patients oxygenation difficulty and hemodynamic instability might ensue. Neonates with postoperative impairment in LV systolic performance, particularly those weighing less than $1000 \mathrm{~g}$, were more likely to have low SAP and abnormal base deficit and need cardiotropes. These changes in myocardial performance are likely to be a consequence of altered loading conditions because they paralleled major alterations in LV exposed vascular resistance and preload, which have important implications when considering the most appropriate cardiovascular intervention. We identified a nonsignificant trend toward a change from in the preload-independent, afterload-adjusted method of assessing.

LV contractility by 8 hours in neonates weighing less than $1000 \mathrm{~g}$. The purpose of this study was not to challenge the decision to perform a PDA ligation but to characterize important changes in cardiovascular physiology. These might facilitate earlier identification of at-risk neonates and more focused postoperative intensive care.

\section{References}

1. Dollberg S, Lusky A, Reichman B. Patent ductus arteriosus, indomethacin and necrotizing enterocolitis in very low birth weight infants: a population-based study. J Pediatr Gastroenterol Nutr. 2005;40:184-8.
2. Ellison RC, Peckham GJ, Lang P, Talner NS, Lerer TJ, Lin L, et al. Evaluation of the preterm infant for patent ductus arteriosus. Pediatrics. 1983;71: 364-72.

3. Teixeira LS, McNamara PJ. Enhanced intensive care for the neonatal ductus arteriosus. Acta Paediatr. 2006;95:394-403.

4. Chorne N, Leonard C, Piecuch R, Clyman RI. Patent ductus arteriosus and its treatment as risk factors for neonatal and neurodevelopmental morbidity. Pediatrics. 2007;119:1165-74.

5. Kabra NS, Schmidt B, Roberts RS, Doyle LW, Papile L, Fanaroff A. Neurosensory impairment after surgical closure of patent ductus arteriosus in extremely low birth weight infants: results from the Trial of Indomethacin Prophylaxis in Preterms. J Pediatr. 2007;150:229-34.

6. Trus T, Winthrop AL, Pipe S, Shah J, Langer JC, Lau GY. Optimal management of patent ductus arteriosus in the neonate weighing less than 800 g. J Pediatr Surg. 1993;28:1137-9.

7. Moin F, Kennedy KA, Moya FR. Risk factors predicting vasopressor use after patent ductus arteriosus ligation. Am J Perinatol. 2003;20:313-20.

8. Teixeira LS, Shivananda SP, Stephens D, Van Arsdell G, McNamara PJ. Postoperative cardiorespiratory instability following ligation of the preterm ductus arteriosus is related to early need for intervention. J Perinatol. 2008;28: 803-10.

9. Taylor AF, Morrow WR, Lally KP, Kinsella JP, Gerstmann DR, deLemos RA. Left ventricular dysfunction following ligation of the ductus arteriosus in the preterm baboon. J Surg Res. 1990;48:590-6.

10. Rowland DG, Gutgesell HP. Noninvasive assessment of myocardial contractility, preload, and afterload in healthy newborn infants. Am J Cardiol. 1995;75: 818-21.

11. Noori S, Friedlich P, Seri I, Wong P. Changes in myocardial function and hemodynamics after ligation of the ductus arteriosus in preterm infants. J Pediatr. 2007; 150:597-602.

12. Stevens B, Johnston C, Petryshen P, Taddio A. Premature Infant Pain Profile: development and initial validation. Clin J Pain. 1996;12:13-22.

13. Smallhorn JF, Gow R, Olley PM, Freedom RM, Swyer PR, Perlman M, et al. Combined noninvasive assessment of the patent ductus arteriosus in the preterm infant before and after indomethacin treatment. Am J Cardiol. 1984;54: 1300-4.

14. Silverman NH, Schiller NB. Cross sectional echocardiographic assessment of cardiac chamber size and ejection fraction in children. Ultrasound Med Biol. 1984; 10:757-69.

15. Colan SD, Borow KM, Neumann A. Left ventricular end-systolic wall stress-velocity of fiber shortening relation: a load-independent index of myocardial contractility. J Am Coll Cardiol. 1984;4:715-24.

16. Barlow AJ, Ward C, Webber SA, Sinclair BG, Potts JE, Sandor GG. Myocardia contractility in premature neonates with and without patent ductus arteriosus. Pediatr Cardiol. 2004;25:102-7.

17. Gill AB, Weindling AM. Echocardiographic assessment of cardiac function in shocked very low birthweight infants. Arch Dis Child. 1993;68:17-21.

18. Walther FJ, Siassi B, King J, Wu PY. Echocardiographic measurements in normal preterm and term neonates. Acta Paediatr Scand. 1986;75:563-8.

19. Pladys P, Wodey E, Beuchee A, Branger B, Betremieux P. Left ventricle output and mean arterial blood pressure in preterm infants during the 1st day of life. Eur J Pediatr. 1999; 158:817-24.

20. Northern Neonatal Nursing Initiative. Systolic blood pressure in babies of less than 32 weeks gestation in the first year of life. Arch Dis Child Fetal Neonatal Ed. 1999;80:F38-42.

21. Kimball TR, Ralston MA, Khoury P, Crump RG, Cho FS, Reuter JH. Effect of ligation of patent ductus arteriosus on left ventricular performance and its determinants in premature neonates. J Am Coll Cardiol. 1996;27:193-7.

22. Lindner W, Seidel M, Versmold HT, Dohlemann C, Riegel KP. Stroke volume and left ventricular output in preterm infants with patent ductus arteriosus. Pediatr Res. 1990;27:278-81.

23. Igarashi $\mathrm{H}$, Shiraishi $\mathrm{H}$, Endoh $\mathrm{H}$, Yanagisawa $M$. Left ventricular contractile state in preterm infants: relation between wall stress and velocity of circumferential fiber shortening. Am Heart J. 1994;127:1336-40.

24. Crepaz R, Pitscheider W, Radetti G, Gentili L. Age-related variation in left ventricular myocardial contractile state expressed by the stress velocity relation. Pediatr Cardiol. 1998;19:463-7.

25. Anderson PA. The heart and development. Semin Perinatol. 1996;20:482-509.

26. McCurnin DC, Yoder BA, Coalson J, Grubb P, Kerecman J, Kupferschmid J, et al. Effect of ductus ligation on cardiopulmonary function in premature baboons. Am J Respir Crit Care Med. 2005;172:1569-74. 